\title{
Couples « linguistiquement mixtes » : une nouvelle catégorie?
}

Gabrielle Varro

\section{(2) OpenEdition \\ 1 Journals}

Édition électronique

URL : http://journals.openedition.org/esp/1160

DOI : 10.4000/esp. 1160

ISSN : 2532-0319

Éditeur

Centre d'Information sur l'Éducation Bilingue et Plurilingue

Édition imprimée

Date de publication : 1 juin 2017

Pagination : 93-100

ISSN : 1127-266X

\section{Référence électronique}

Gabrielle Varro, « Couples « linguistiquement mixtes » : une nouvelle catégorie ? », Éducation et sociétés plurilingues [En ligne], 42 | 2017, mis en ligne le 01 février 2018, consulté le 20 avril 2019. URL : http:// journals.openedition.org/esp/1160 ; DOI : 10.4000/esp.1160 


\section{COUPLES «LINGUISTIQUEMENT MIXTES», UNE NOUVELLE GATÉGORIE?}

\section{Gabrielle VARRO}

Oggi, in Francia come altrove, in occasione dei contatti sempre piu frequenti fra persone provenienti da orizzonti diversi - che si tratti di cittadini di paesi distinti o di concittadini di regioni diverse di uno stesso paese che non parlano la stessa lingua nelle rispettive famiglie d'origine - si formano coppie. Alcuni ricercatori si sono recentemente interessati a questa nuova categoria di coppia, passibile di essere allargata al concetto di intersezionalità (combinazione di risorse): le coppie linguisticamente miste.

Parole chiave: mescolanza linguistica, coppia mista, trasmissione, nozione di differenza, intersezionalità, categoria sociologica

Today, in France as elsewhere, more and more people who didn't speak the same language when they were growing up - whether from different countries or from different regions of the same country - meet, form couples and raise new families. «Linguistically mixed couples» have recently attracted the attention of research scholars, enlarging the category of conjugal mixedness which emerges as a new sort of intersectionality (combination of resources).

Key-zords: linguistic mixedness, mixed couple, transmission, idea of difference, intersectionality, category

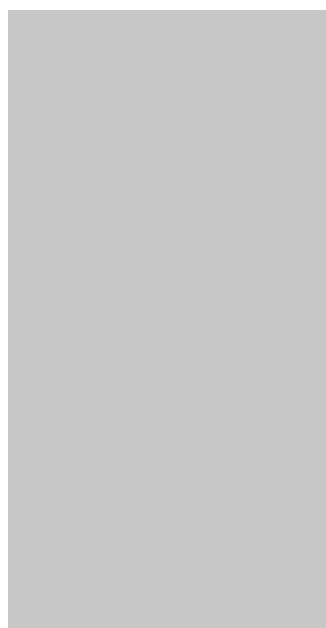

ujourd'hui, en France comme ailleurs, des couples se for-
ment lors des contacts de plus en plus fréquents entre per-
sonnes venues d'horizons différents, qu'il s'agisse de citoyens
de pays distincts ou de concitoyens issus de régions différentes qui ne
parlaient pas la même langue dans leur famille d'origine. Des cher-
cheurs se sont assez récemment intéressés à cette nouvelle sorte de
couple: les couples «linguistiquement mixtes».
Naturellement, on peut affirmer que tout couple est mixte puisque
composé de deux individus distincts, qui ont connu des socialisations
dans des lieux différents. Cependant, la «mixité conjugale» a inspiré
des recherches sur toutes les sortes de rencontres qui, sur un plan
individuel ou sociétal, sont supposées problématiques, depuis les
mariages inter-religieux, rapidement concurrencés par les unions
inter-raciales, puis inter-ethniques, enfin internationaux (Cottrell
1997).
Sous l'angle de la confrontation au réel, on peut se féliciter que les
recherches sur la mixité matrimoniale s'affinent de plus en plus et 


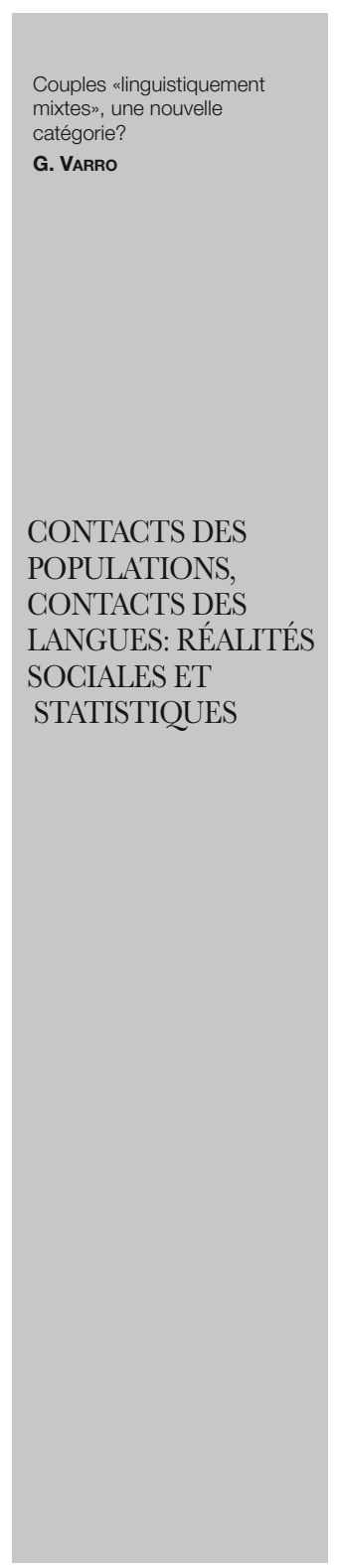

couvrent de plus en plus de champs et de plus en plus de pays (Langage et Société n¹47, 2014). Après les couples dits mixtes parce qu'internationaux, l'élargissement de la catégorie a plus récemment débouché sur les couples «linguistiquement mixtes»; ceci ne signifie pas qu'on ne tenait pas compte auparavant de la différence de langues entre conjoints, mais là où elle n'était qu'un parmi d'autres facteurs de différentiation (comme la religion ou la nationalité), elle a pu être érigée en variable principale par rapport à laquelle analyser des comportements et tirer des conclusions (Zeiter, sous presse).

Le présupposé de ces recherches et de ces catégorisations - ou plutôt ce qu'elles nous montrent - est que dans nos sociétés, même les plus modernes, la norme de l'homogamie domine toujours les esprits et (certaines) institutions (telles que les religions) et concurremment, que le fait d'y contrevenir ne peut qu'être source de problèmes.

Vus sous cet angle, on peut dire que les mariages internationaux impliquent des situations plus «neutres», non spécialement problématiques: c'est le sens adopté par les institutions de la statistique (Ined, Insee) pour lesquelles «mariage mixte» désigne un couple composé d'un-e citoyen-ne et d'un-e étranger-e, sans introduire de jugement de valeur quant aux différences en présence. Quand un tel jugement de valeur est sous-jacent, le couple est souvent désigné comme «interculturel». Cette observation m'amène à penser que la notion d'«interculturel» suppose elle-même un jugement de valeur qui renvoie à un rapport inégalitaire, de dominant à dominé. En effet, à y regarder de près, l'interculturel implique une rencontre de cultures différentes et il est très rare qu'elles ne soient hiérarchisées entre elles: culture-langue majoritaire $v s$. culture-langue minoritaire, culturelangue dominante vs. culture-langue dominée. Ce sont des réalités sociales la langue-culture du pays de résidence est majoritaire chez le couple mixte résidant au pays du conjoint citoyen) qui risquent de devenir aussi des réalités symboliques la langue-culture du conjoint transplanté est par définition minoritaire et risque la domination).

Dans cette optique, la notion de «différence culturelle» se trouve affectée d'un coefficient négatif qui ne fait qu'exprimer des attitudes souterraines de xénophobie, protectionnistes et chauvins, voire racistes, qui demeurent tapies et occultées au fond des rapports humains. Il semble pratiquement impossible de dépasser le clivage «nous/eux», dont le signe distinctif reste la notion de «différence» entre les individus et la suspicion qu'elle engendre.

En nous inspirant de François Jullien (2016), soulignons qu'ériger les «différences culturelles» des conjoints en critères principaux de la mixité conjugale serait une erreur: dire «différent» sépare et oppose, alors que se positionner par rapport aux écarts entre les ressources cul- 


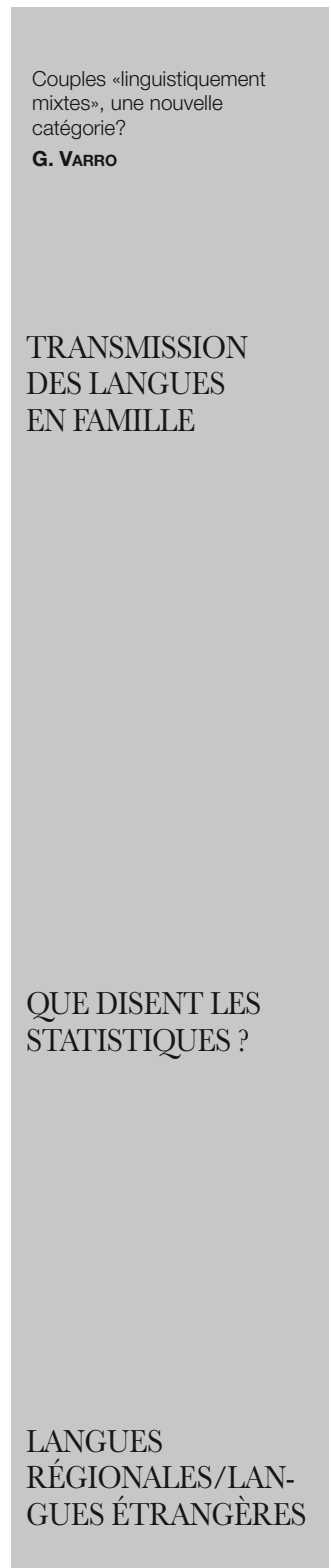

turelles que chacun apporte à la vie commune permet de considérer l'ensemble sans effectuer de comparaison ou d'opposition.

Ainsi, pour les couples mixtes, dont un des conjoints «vient d'ailleurs», le défi principal consiste à établir et maintenir, au propre comme au figuré, dans la réalité comme dans le symbolique, une égalité entre les deux partenaires, c'est-à-dire une égale valorisation, ou une juste appréciation des ressources respectives. Car il est clair que l'égalité ne peut pas se concevoir terme à terme.

Des points établis par des recherches de terrain peuvent être vérifiés par la statistique. L'observation, par exemple, que les couples mixtes transmettent le moins la langue étrangère (minoritaire, minorisée) aux enfants (Platiel 1987, Heredia-Deprez 1989). En effet, il est fréquent qu'un couple mixte adopte comme langue commune la langue du conjoint majoritaire - la langue du pays de résidence - d'autant plus quand celui-ci ne parle pas celle du conjoint. En France (comme ailleurs), les couples mixtes transmettent donc davantage la langue nationale, officielle (le français) que la langue du conjoint migrant.

Des recherches de terrain ont également montré que pères et mères ne transmettent pas les langues de la même façon (Deprez \& Dreyfus 1998). En outre, des conjoints interrogés séparément sur leurs pratiques langagières n'en ont pas toujours la même vision. Ceci montre que la déclaration des intéressés quant aux pratiques langagières de leur famille ou de leur entourage est subjective et ne peut se passer de l'observation directe par le chercheur (dont la subjectivité est aussi en jeu, bien entendu ....).

Les enquêtes menées par les instituts de statistiques (Ined, Insee), parallèlement aux recensements de la population, livrent des résultats à grande échelle sur les pratiques et la transmission des langues en France (Héran et alii. 2002, Clanché 2002). L'étude Familles (INED 1999) a touché 380,000 personnes et a fait apparaître pas moins de 6,700 intitulés de langues et de parlers en France. Elle «confirme la domination indiscutable du français en France, ainsi que l'usage accru des langues apprises à l'école, mais/elle/révèle aussi la richesse du patrimoine linguistique lié à la diversité de nos origines et de nos expériences. Le français vient couronner cet héritage; il ne l'abolit pas» (Héran et alii. 2002: 4).

À la différence des langues étrangères, la plupart des langues régionales en France ont été transmises seulement de façon occasionnelle, en accompagnement du français, et souvent par un seul parent. C'est particulièrement vrai de la langue d'oc et des langues d'oill, déjà en 


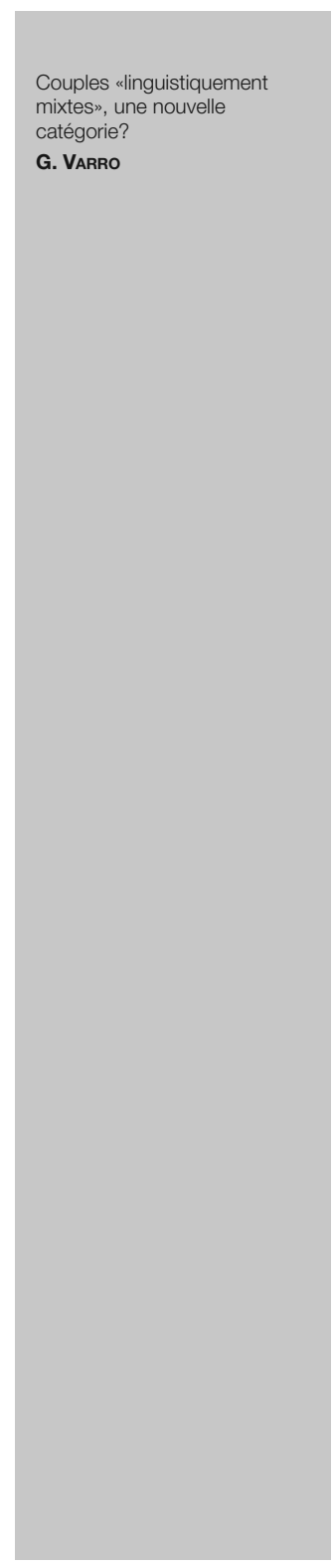

fort recul dans l'ancienne génération. Il n'en demeure pas moins que ces langues ont accompagné l'enfance de très nombreux adultes actuellement encore en vie. Reçu plus souvent sur un mode habituel (660,000 personnes) qu'occasionnel $(240,000)$, l'alsacien était encore il y a une génération la langue régionale la mieux transmise (pour une actualisation de la situation de l'alsacien, of. Huck 2015). De même, une bonne partie de la Moselle voisine se transmettait usuellement en famille un parler francique, le platt lorrain. Dans le reste de la France, seul le basque a connu une situation similaire.

La pratique déclarée des langues régionales est donc dans l'ensemble peu répandue en France (un tiers des adultes seulement disent avoir retransmis la langue familiale).

Concernant les déclarations des pratiques langagières des couples mixtes franco-étrangers, on constate que, lorsque l'homme est le migrant du couple, c'est la transmission de l'arabe et de l'italien qui domine le tableau. C'est le cas de l'espagnol et de l'allemand lorsque la femme est la migrante du couple. Les couples français (non-mixtes) se signalent par la transmission de l'anglais, les couples migrants nonmixtes (i.e. conjoints du même pays) par la transmission de l'arabe et du portugais et les couples mixtes migrants (de deux pays différents) par celle de l'arabe et de l'espagnol.

L'arabe (ou arabe + berbère $=33 \%, c f$. Filhon 2009) a été déclaré par près de $30 \%$ des couples migrants (du même pays ou de pays différents), ce qui est un chiffre remarquable, étant donné que la plupart des auteurs avaient jusqu'ici conclu à la perte ou l'absence de l'arabe parmi les enfants de migrants.

Contrairement aux langues étrangères, dont toutes était davantage citées par les femmes que par les hommes, on remarque pour les langues régionales une alternance entre celles qui sont citées plus par les hommes et celles citées plus par les femmes. Les femmes sont plus nombreuses à déclarer qu'elles transmettent l'alsacien, une langue d'oc, le breton, une langue d'oil, le platt, le créole ou le basque, les hommes disent davantage transmettre le catalan, le corse et le francoprovençal.

En fait, qu'il s'agisse de couples «mixtes» ou non, toutes les langues ont été davantage citées par les femmes, sauf le catalan, le corse et le francoprovençal, cités majoritairement par des hommes.

Cependant, la confrontation entre études qualitatives et quantitatives doit inciter à examiner de plus près ce que "transmettre" veut dire réellement, outre une déclaration d'intention de la part de parents désireux de transmettre une langue... Il s'agit bien de la perception de faits sociaux (ici, la réalité de la transmission) et non d'un fait avéré de façon incontestable. 


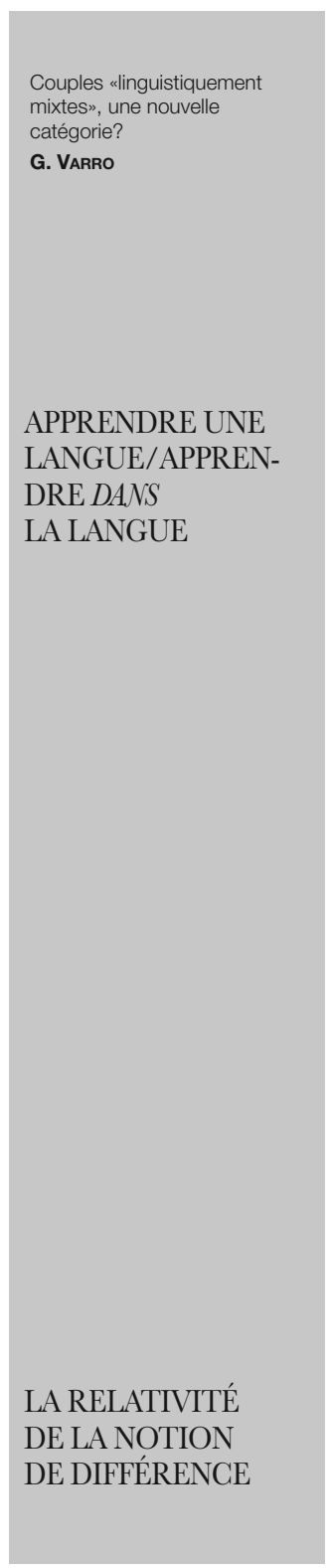

La seconde grande question posée par l'enquête de l'INED concerne le rôle de l'école dans la pratique des langues minoritaires et elle reçoit une réponse assez tranchée: cette influence est nulle, voire négative, s'il s'agit d'apprendre la langue; elle est au contraire source de "bilingualisation" si on apprend dans la langue.

Si dans le recensement, l'apprentissage scolaire paraissait primordial pour la vie d'une langue minoritaire, sur le terrain, il recule au second plan. Par contre, concernant la langue basque, par exemple, on obtient un aperçu de ce que l'immersion précoce totale peut produire selon l'âge des apprenants et le contexte de leur apprentissage. Les Calandretas, «écoles laïques associatives (loi de 1901), proposent un enseignement bilingue en occitan et français, de la maternelle au CM2». La langue principale de l'école est l'occitan, le français étant enseigné comme une matière parmi d'autres. «L'occitan est donc dominant ou voulu comme tel dans la classe mais on constate que le français redevient prépondérant dès le passage du seuil de la cour de récréation», cette "diglossie" étant renforcée à l'extérieur de l'école et notamment avec le passage au collège, où les ex-Calandrons cachent même souvent leur connaissance de la langue minoritaire («la "vergogna" sans doute», comme dit un parent (Dompmartin-Normand 2002).

L’influence de l'école sur la pratique de la langue régionale reçoit ici une confirmation vigoureuse: l'enseignement en occitan est productif, l'enseignement de l'occitan est totalement contre-productif. À tel point que l'auteur recommande qu'«en absence de moyens réels d'enseigner «en» occitan au collège, peut-être vaudrait-il mieux laisser ces collégiens bilingues sur leurs bons souvenirs de l'immersion et miser sur un renouveau d'intérêt pour la langue minorée à l'entrée à l'université, qui serait attesté dans des situations similaires (enquête en cours au Val d'Aoste)» (voir aussi Decime, 2002).

Des études de cas dans des pays différents débouchent sur des conclusions similaires: du point de vue sociologique et linguistique, il est clair que les données proprement humaines du terrain (statuts des langues, attitudes des locuteurs, habitudes prises, etc.) sont plus déterminantes que toutes les planifications et réformes éducatives mises en œuvre; du point de vue statistique, tant que l'on ne leur restitue pas la complexité des réalités du terrain, les chiffres ne sont qu'apparence.

En adoptant un point de vue diachronique, on constate que les recherches sur les couples mixtes sont tributaires des préoccupations d'une époque, autrement dit de «modes»; définis par leurs appartenances de classe, des conjoints réunissant un aristocrate et une roturière sont considérés comme «mixtes», ainsi qu'un patron d'industrie 


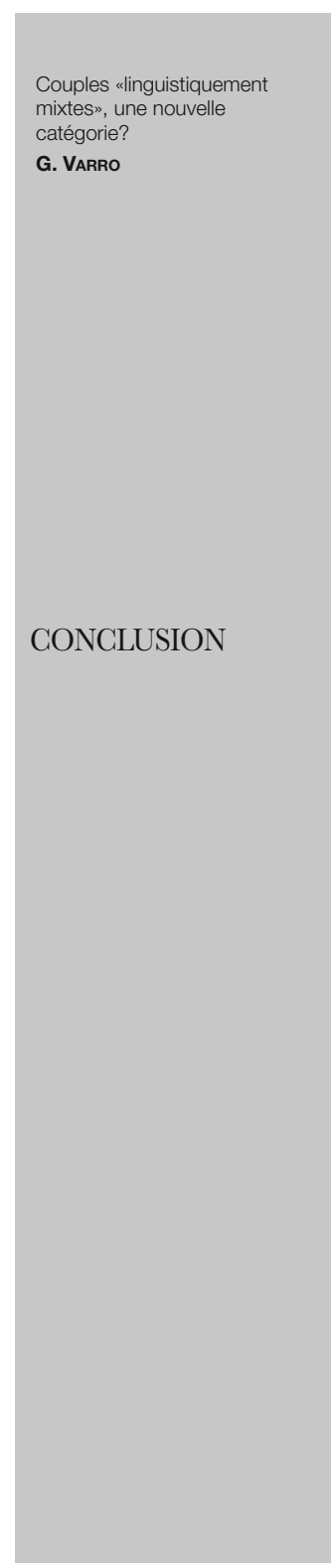

et sa secrétaire - on disait «mésalliance» autrefois ( $c$. Varro 2003 chapitre 1) - et le terme n'est pas inconnu chez les grands bourgeois d'aujourd'hui étudiés par les Pinçon-Charlot (1999).

Un point de vue diachronique ferait ressortir que certaines observations faites depuis longtemps dans la littérature sociolinguistique comme dans les enquêtes quantitatives se confirment réciproquement: la langue minoritaire en position dominée est vouée à disparaitre du répertoire familial; cette langue inspire de la nostalgie chez les individus et possède une valeur symbolique. En Bretagne comme en Alsace ou ailleurs, le français est devenu la «langue incontournable de la modernité». Mais pour en rester à l'exemple des langues minoritaires, on retrouve au sein même d'un Etat-nation comme la France des appartenances régionales qui, lorsque par exemple un-e bascophone bilingue avec le français se trouve en couple avec un-e français-e qui ne parle pas le basque (Lacroix 2014), peuvent être interprétées en termes de «mixité conjugale linguistique».

Aujourd'hui, l'approche sociologique qui paraît la plus à même d'aborder les réalités complexes individuelles et collectives de nos sociétés est «l'intersectionnalité». Popularisé par les travaux de la juriste afro-américaine Kimberlé Crenshaw dans les années 1980-90, le concept propose une nouvelle vision de la pluralité des minoritésqu'elles soient définies par des critères de genre, race, sexualité, classe, voire même âge ou handicap - en se plaçant aux points d'intersection des catégories (Matsuda et alii. 1993). L'«intersectionality» désigne la situation de personnes subissant simultanément plusieurs formes de domination ou de discrimination dans une société (par exemple, être femme et noire - et homosexuelle - aux Etats-Unis; étrangère ou immigrée - et voilée - en France...).

Le concept de mixité, pris au sens individuel comme dans le cas d'un enfant de couple mixte, permet d'appréhender la situation spécifique d'une personne ou d'un couple en société. Comme suggéré plus haut, la mixité n'est pas un affrontement de «différences» mais une combinaison de ressources. Dans chaque couple, on observe des tiraillements entre les partenaires; l'épreuve de forces, déjà mise en scène il y a 33 ans dans La femme transplantée (Varro, 1984) est toujours à chaque fois décortiquée à nouveau.

L'analyse du fonctionnement des couples peut s'appuyer sur l'étude de leur mixité linguistique mais les autres angles d'attaque restent possibles - aborder les réalités vécues des couples tout à la fois par la position de majoritaire ou minoritaire de l'un-e ou l'autre partenaire, par le fait d'être homme ou femme, migrant ou autochtone, par le choix des prénoms des enfants ou par le choix de lieu de vie, 


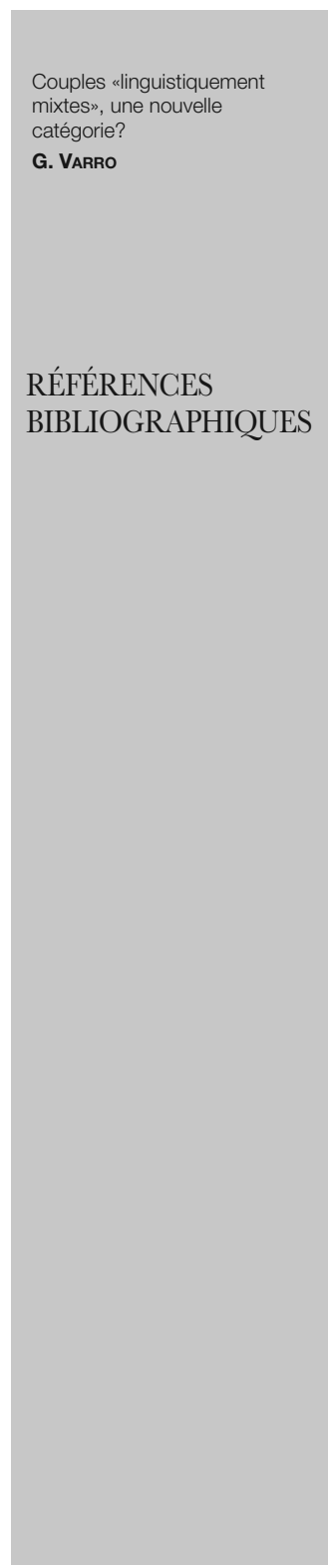

débouche sur un tableau où tout semble à la fois spécifique et général: les couples décrits sont particuliers par les traits qu'eux-mêmes ou les chercheurs mettent en exergue et identifient comme «culturels» et «différents», mais simultanément ils sont «ordinaires» dans leur diversité même.

La mixité peut ainsi être vue comme une «intersectionnalité positive», en ce qu'elle combine les ressources des deux personnes en regard sans les opposer mais en maintenant par là leur intérêt et leur désir mutuels en éveil.

CLANCHÉ F. 2002. Langues régionales, langues étrangères: de l'héritage à la pratique, Insee-Première, nº 830 (février).

DECIME R. 2002. Regards croisés sur l'éducation bilingue en Vallée d'Aoste, Éducation et sociétés plurilingues, $\mathrm{n}^{\circ} 13$ (décembre).

DEPREZ C., DREYFUS M. 1998. Transmission et usages des langues. Couples mixtes à Paris et à Dakar, pp. 201- 228 in PHILIPPE C., G. VARRO, G. NEYRAND (éds.). Liberté, Egalité, Mixité... conjugales. Une Sociologie du couple mixte. Paris, Anthropos.

DOMPMARTIN-NORMAND G. 2002. Collégiens issus de Calandreta: quelles représentations de l'occitan? Langage et Société $\mathrm{n}^{\circ} 101$ (septembre): $35-54$.

FILHON A. 2009. Langues d'ici et d'ailleurs: Transmettre l'arabe et le berbère en France, Paris, INED.

HERAN F., FILHON A., DEPREZ C. 2002. La dynamique des langues en France au fil du XXe siècle, Population E̊ sociétés, n³76 (février).

HEREDIA-DEPREZ C. de. 1989. Le plurilinguisme des enfants à Paris. Revue Européenne des Migrations Internationales 5, 2: 71-87.

HUCK D. 2015. Une histoire des langues de l'Alsace. Strasbourg, La nuée bleue.

INED. 1999. Etude de l'Histoire Familiale et Recensement de la population française.

JULLIEN F. 2016. Lidentité culturelle n'existe pas. Paris, Herne.

LACROIX I. 2014. Valeur symbolique de la langue au Pays basque français et choix de l'école pour les enfants de couples linguistiquement mixtes, Langage et Société ${ }^{\circ} 147$ (mars): 67-82.

Langage et Société ${ }^{\circ} 147$ (mars). Familles plurilingues dans le monde. Mixités conjugales et transmission des langues, Dossier. B. COLLET, C. DEPREZ, G. VARRO (éds.).

MATSUDA M. J., LAWRENCE III G. R., DELGADO R., WILLIAMS CRENSHAW K. 1993. Words that Wound: Critical Race Theory, Assaultive Speech and the First Amendment, Westview Press. PINÇON M., CHARLOT M. 1997. Voyage en grande bourgeoisie, 


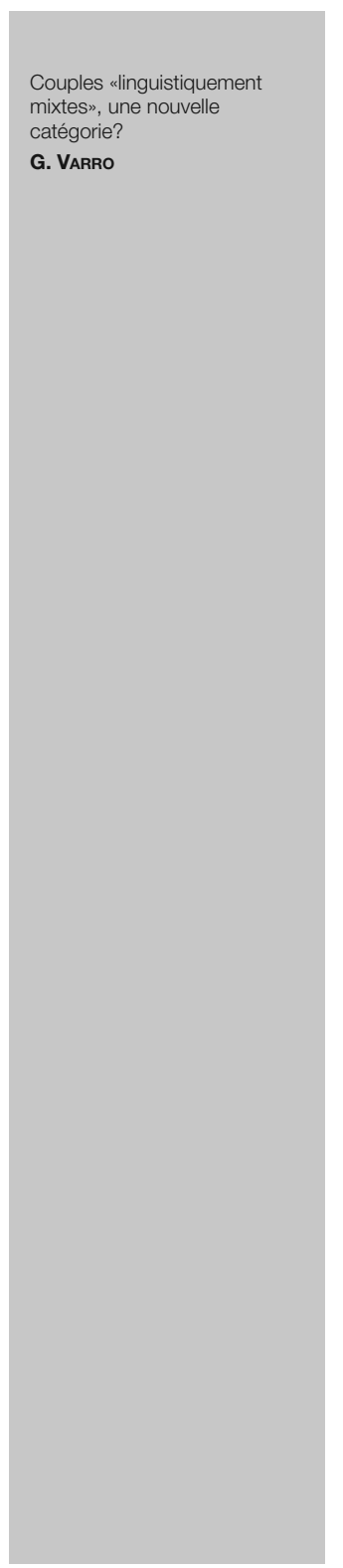

Paris, PUF.

PLATIEL S. 1988. Les langues d'Afrique Noire en France. Des langues de culture face à une langue de communication, pp.9-30 in VERMES G. (dir.), Vingt-cinq communautés linguistiques de la France. 2 vols. Paris, L'Harmattan, vol. 2.

VARRO G. 1984. La femme transplantée. Une étude du mariage franco-américain et le bilinguisme des enfants. Lille: Presses du Septentrion.

VARRO G. 2003. Sociologie de la mixite. De la mixité amoureuse aux mixités sociales et culturelles. Paris: Belin.

ZEITER A.-C. Sous presse. Dans la langue de l'autre. Se construire en couple linguistiquement mixte. Lausanne, ENS Editions. 